\title{
Diurnal effects of motor activity and fatigue in Parkinson's disease
}

\author{
J J van Hilten, G Hoogland, E A van der Velde, H A M Middelkoop, G A Kerkhof, \\ R A C Roos
}

\begin{abstract}
Wrist motor activity was monitored continuously in 65 patients with Parkinson's disease (PD) to assess the influence of disease severity and excessive fatigue on the diurnal motor activity pattern. Mildly or moderately affected PD patients had a similar diurnal pattern to that of 68 healthy controls, with a late morning peak; however, mean levels of motor activity were lower. The most severely affected patients showed an overall flattened diurnal pattern. Results refute the existence of end of day deterioration, but instead suggest a "depressed morning start" in the most severely affected patients with PD. Excessive fatigue was not reported at a particular time of day and did not influence the diurnal motor activity pattern.
\end{abstract}

$(\Im$ Neurol Neurosurg Psychiatry 1993;56:874-877)

During wakefulness, healthy human subjects exhibit a distinct pattern of motor activity characterised by a rise within the first hours after awakening, a stable level during the remaining part of the morning, and a gradual decrease in the afternoon and evening. ${ }^{123}$

Parkinson's disease (PD) is characterised by an initially stable response to levodopa treatment. After 5 years of levodopa treatment, about half of the patients with PD experience fluctuations in motor performance. ${ }^{4-6}$

However, regardless of these response fluctuations, patients with PD may report a decline of functional performance and increasing fatigue as the day progresses. ${ }^{4-7}$

It is unclear whether this end of day deterioration in PD really exists. In the present study we assessed the diurnal motor activity patterns in patients with PD, and determined whether the disease severity and presence of excessive fatigue had any influence upon this.

\section{Patients and methods}

We studied 65 patients with idiopathic PD (table) and 68 healthy subjects (26 men, 42 women; mean age (SD) 66.9 (11.4) years, range $36-83$ years). All patients and control subjects had a mini-mental state examination score above $24 .^{8}$

All patients and subjects were interviewed and examined by one of us (JvH). The control subjects were healthy and independent.
They received a routine neurological examination at home. All patients were assessed by the Hoehn and Yahr (H\&Y) and Unified Parkinson's Disease Rating Scale (UPDRS)..$^{10}$ Forty five patients used levodopa (in combination with a peripheral decarboxylase inhibitor); 20 showed a stable response with levodopa, 25 patients suffered from motor fluctuations (wearing off $n=16$; unpredictable off periods $n=$ four; both $n=$ five). Four patients reported mild early morning dystonia of one or both feet. As we have shown previously, PD patients suffering from on off fluctuations with choreiform dyskinesias may show a typical diurnal activity pattern which is characterised by a regular or irregular switching of periods with low and high activity. ${ }^{3}$ The low activity periods represent the "off state" with severe hypokinesia and rigidity. The high activity periods represent the "on state" with choreiform dyskinesias. In this study the diurnal activity pattern was used to evaluate the presence of end of day deterioration as well as to study the possible influence of excessive fatigue upon this. Patients with on off fluctuations have a drug induced diurnal activity pattern in which the levodopa induced "high activity" periods occur throughout the day. In view of the issue addressed in this study, the diurnal activity patterns of these patients are not suitable for study. Consequently, five PD patients who suffered from generalised choreiform dyskinesias were excluded from this study.

We defined fatigue as a sense of physical tiredness and lack of energy, interfering with physical functioning and social life, distinct from mental exhaustion, sadness, sleepiness, and impaired motor function secondary to PD symptoms (weakness, stiffness, and tremor). To assess the seriousness of fatigue

Table Characteristics of 65 patients with idiopathic Parkinson's disease.

\begin{tabular}{lrrc}
\hline Clinical variable & Mean & \multicolumn{1}{c}{$S D$} & Range \\
\hline Current age (years) & $66 \cdot 6$ & $9 \cdot 9$ & \multicolumn{1}{c}{$42-83$} \\
Age of onset (years) & $60 \cdot 5$ & $10 \cdot 0$ & $40-76$ \\
Duration of illness (years) & $6 \cdot 1$ & $4 \cdot 7$ & $0 \cdot 5-21$ \\
Duration of levodopa & $3 \cdot 4$ & $4 \cdot 6$ & $0-18$ \\
$\quad$ treatment (years) & $0 \cdot 3$ & $0 \cdot 4$ & $0-1 \cdot 6$ \\
Dosage of levodopa (g/day) & $2 \cdot 9$ & $0 \cdot 8$ & $1-5$ \\
H \& Y stage (off) & $38 \cdot 8$ & $16 \cdot 2$ & $10-87$ \\
UPDR total score (off) & $13 \cdot 3$ & $7 \cdot 6$ & $1-36$ \\
UPDRS ADL score (off) & & & \\
UPDRS motor examination & & & \\
$\quad$ score, dominant side /off & $21 \cdot 1$ & $8 \cdot 2$ & $5-39$ \\
UPDRS Motor examination & & & \\
$\quad$ score (non-dominant side/off) & $20 \cdot 1$ & $8 \cdot 2$ & $5-40$ \\
\hline
\end{tabular}

H\&Y = Hoehn and Yahr; UPDRS = unified Parkinson's disease rating scale; $\mathrm{ADL}=$ activities of daily living. 
we used a 5 point rating scale ( $1=$ absent, $2=$ sometimes present, $3=$ often present, $4=$ very often present, $5=$ continuously present). Only patients with excessive fatigue (score $\geq 3$ ), were questioned further about the time this symptom mainly occurred. We considered excessive fatigue as a disease related symptom and control subjects who suffered from excessive fatigue were excluded.

The present study aimed to evaluate fatigue as a symptom of PD. However, in PD depression is frequently encountered and fatigue may also occur as a symptom of the depression. Therefore, we tried to exclude PD patients with prominent clinical signs of depressive effect. Depression was assessed by a Dutch translated and validated version of the Montgomery-Åsberg depression scale. ${ }^{11}$ It should be noted that it is often impossible to separate depression from primary symptoms of PD. The problem lies in the assessment of items like fatigue, sleep disturbances, and loss of appetite. These complaints may also occur as PD or medication related symptoms and contaminate the performance. Therefore, our screening procedure, inquiring about symptoms of depression particularly focused on the items regarding sadness, motivation, attention, pessimism, and suicidal thoughts. Using these items an additional four patients were excluded from this study.

Because of the possible influence of daytime sleepiness on our results both groups were evaluated with regard to presence of self-reported daytime sleepiness (assessed with a similar 5-point rating scale). No difference was found between both groups regarding the presence of excessive daytime sleepiness $(p=0 \cdot 2)$. Additionally in the patient group no relationship emerged between excessive daytime sleepiness and the use of dopaminergic drugs.

We measured motor activity with an activity monitor, worn on the non-dominant wrist from Monday $7 \mathrm{pm}$ to Sunday $11 \mathrm{am}$. Although asymmetry of signs and symptoms in $\mathrm{PD}$ is not uncommon, we selected the non-dominant arm in all patients. Measurements of motor activity of this arm reflect total body movement much better because the dominant arm is more involved in performing specific tasks. ${ }^{12}$ Subjects maintained their habitual 24 hour pattern of activities, and removed the monitor only when taking a bath. During the recording period all subjects kept a log. They recorded the time they switched off the light to go to sleep, the time of definitive awakening, any naps, and the time they removed the monitor when taking a bath.

The characteristics of the activity monitor (Gaehwiler Electronic, $\mathrm{CH}-8634$ Hombrechtikon, Switzerland) used in this study have been reported previously. ${ }^{313}$ In short, the device counts the occurrence of suprathreshold motor activity (accelerations $>0.1 \mathrm{~g}$ with filtering of the analogue sensor signal by a bandpass filter of $0 \cdot 25-3 \mathrm{~Hz}$ over 15 second epochs, and stores the resulting sum as a 1 byte value in a 32 kilobyte solid state memory). After completion of the recording, the data are read out in a personal computer and further analysed.

Healthy subjects have a distinct diurnal pattern of motor activity characterised by four diurnal periods with different activity levels: (1) the first two hours after awakening, (2) the remaining part of the morning till 1300 , (3) the afternoon; $1300-1900$, (4) the evening; $1900 \mathrm{hr}$ till lights out. ${ }^{13}$ Therefore, for each of the four diurnal periods the following parameters were calculated in all subjects:

(1) The activity level, expressed as the mean number of counts per 15 seconds.

(2) The movement index, the percentage of epochs with any movement (activity count $>0$ ), with respect to all 15 second epochs that make up the period. The movement index reflects the proportion of activity and immobility of that period.

\section{Statistical analysis}

To study the influence of the severity of a clinical variable on the diurnal motor activity pattern, we selected the following patient categories:

(1) disease severity:

(A) H\&Y stage:

(a) stage I-II ( $\mathrm{n}=18$; one patient H\&Y stage I with non-dominant side affected),

(b) stage III ( $\mathrm{n}=35)$,

(c) stage IV-V ( $\mathrm{n}=12$; one patient $\mathrm{H} \& \mathrm{Y}$ stage $\mathrm{V})$.

(B) UPDRS total score (range 0-152):

(a) score $10-30(n=20)$,

(b) score $31-50(n=30)$,

(c) score $51-87(n=15)$.

(2) excessive fatigue:

(a) absent; score $\leq 2(n=34)$,

(b) present; score $\geq 3(n=31)$.

We also explored if different classifications based on scores which reflect resting tremor (controlled for hypokinesia), hypokinesia rigidity (controlled for resting tremor), the presence of motor fluctuations, as well as the presence of excessive daytime sleepiness, influenced the results. Since this did not produce different findings, the presentation of the results will be restricted to the main variables mentioned above.

For both patients and controls we examined initially the distribution of the activity level and the movement index of each diurnal period. For both groups the data of each diurnal period were normally distributed.

The results were analysed by using an analysis of variance (ANOVA) procedure (statistic package for Social Science $\mathrm{pc}^{+}$). In the ANOVA a repeated measures design was carried out ${ }^{14}$ the activity parameters are considered as dependent variables. The factor "subjects" is nested within the fixed factor "groups" and crossed with the fixed factor "period". For each clinical variable, analyses were carried out in the following order. Firstly, the pooled patient severity groups and the control group are compared (patients 
versus controls analysis). Secondly, the patient severity groups are compared mutually (between patient analysis). If both between controls versus patients and between patient analysis revealed significant differences, each severity group is compared with the control group to explain which patient group(s) is responsible for these differences. Accordingly, we investigated: differences between groups about the mean values of the activity level and movement index (taken over all periods); the existence of a diurnal pattern for these parameters; and group differences about their diurnal pattern.

We defined end of day deterioration as a diurnal pattern with a statistically significant larger decrease of the activity level and movement index in the afternoon and evening period in patients than controls. $p$ Values $<0.05$ are considered statistically significant.

\section{Results}

The mean time of "lights-out" for the patients $(2346 \mathrm{~h})$ and controls $(2358 \mathrm{~h})$ showed no difference $(p=0.43)$. Similarly, no difference $(p=0.73)$ emerged regarding the mean time of "definitive awakening"; patients $(0719 \mathrm{~h})$, and controls $(0713 \mathrm{~h})$.

\section{DISEASE SEVERITY}

The H\&Y stage III and IV-V group had lower mean values $(p<0.000)$ for the activity level than controls. For the movement index all

Figure 1 The diurnal pattern of the activity level (A) and movement index (B) for the controls and HE Y stage patient groups. Values are given as $a$ mean and $1 S D$.
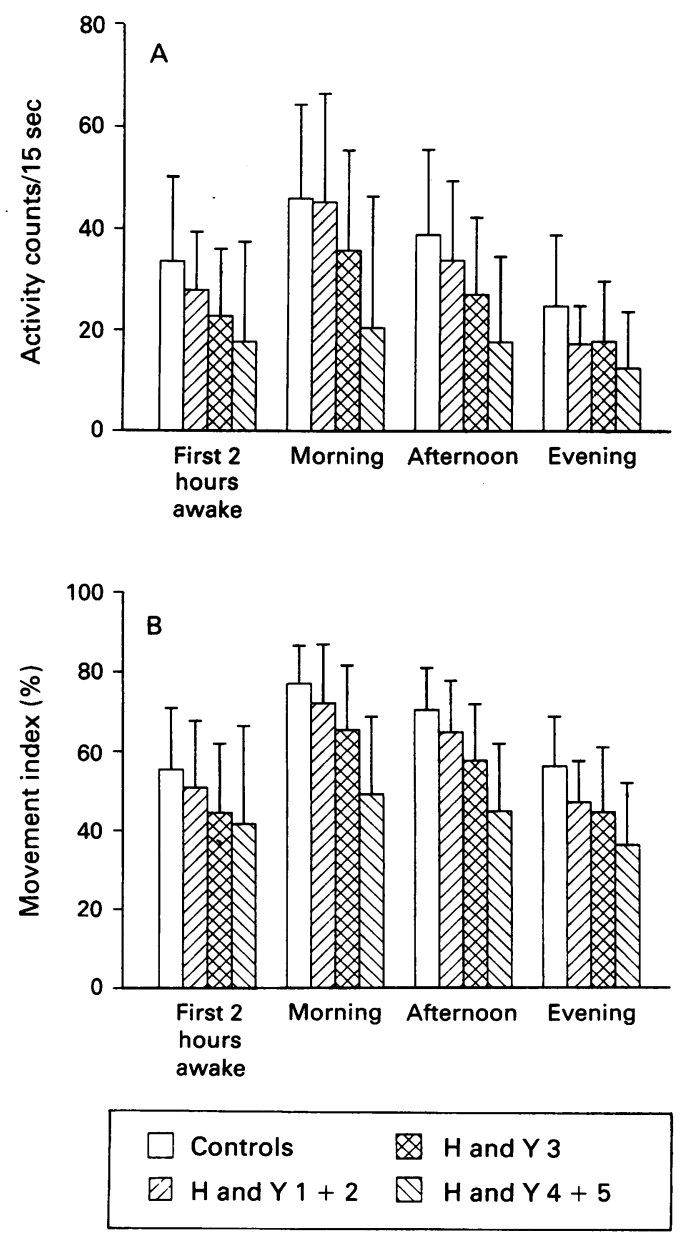

H\&Y stage groups had lower mean values $(p<0.000)$ than the controls. The diurnal pattern of the activity level (AL) and movement index (MI) in the H\&Y stage groups I-II and III was normal, while in the H\&Y stage IV-V group it was severely flattened (fig 1A B); between patient group analysis for the AL: $p=0.004$, and for the MI this was nonsignificant).

All UPDRS total score groups had lower ALs $(p<0.000)$, while no difference was found between the patient groups. Similarly, for the MI in all UPDRS severity groups the values are lower than the controls $(p<0.000)$. The mean level of the MI was inversely proportional to the group severity. Only the most affected group (UPDRS score 51-87) had a severely flattened diurnal pattern of the $\mathrm{AL}$ and $\mathrm{MI}$ index (fig $2 \mathrm{~A} \mathrm{~B}$ ); between patient group analysis for the $A L$ : $p=0.009$, and for the MI: $p=0.016$.

\section{FATIGUE}

Excessive fatigue was present in 31 patients. Most of the patients $(n=14)$ reported no particular time of day at which fatigue mainly occurred. The other patients reported fatigue to be worse during the morning $(n=2)$, noon $(n=5)$, afternoon $(n=5)$, evening $(n=5)$. Control subjects had significantly higher mean values for the activity level and movement index than both PD groups $(p<$ $0 \cdot 000$ ). Compared with controls, patients had a similar diurnal pattern of the activity level
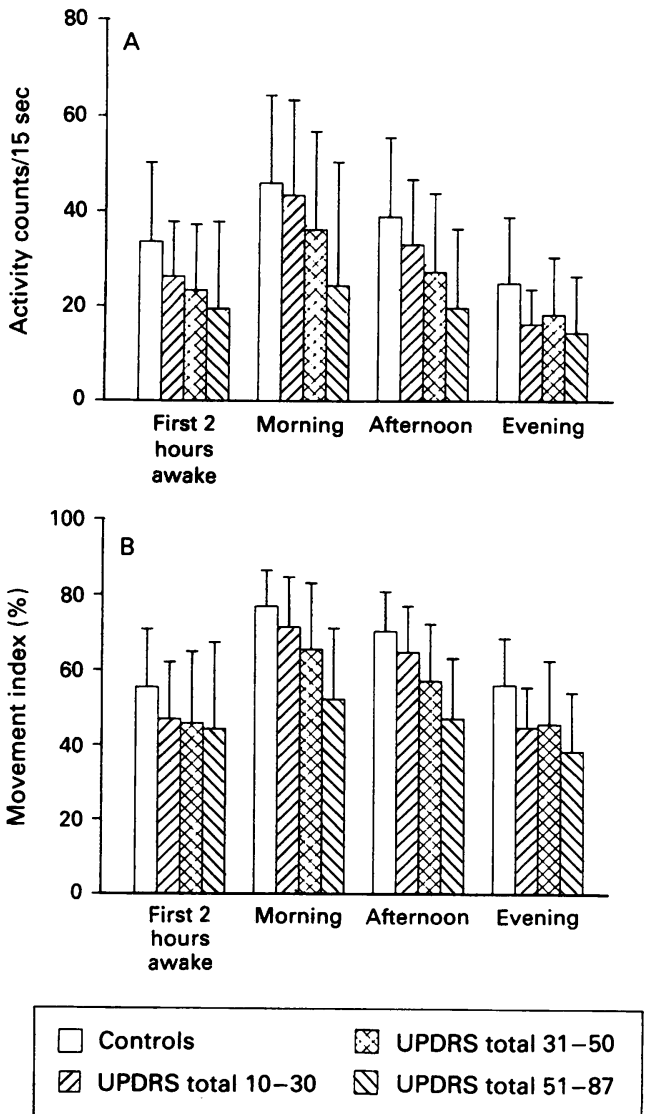

Figure 2 The diurnal pattern of the activity level $(A)$ and movement index $(B)$ for the controls and UPDRS total score groups. Values are given as a mean and $1 S D$. 
Figure 3 The amount of change of the movement index between the consecutive periods of the controls, and the most affected patient groups of the UPDRS total score and HE'Y stage (UPDRS $=$ unified Parkinson's disease rating scale, $H \mathcal{E} Y$ $=$ Hoehn and Yahr).

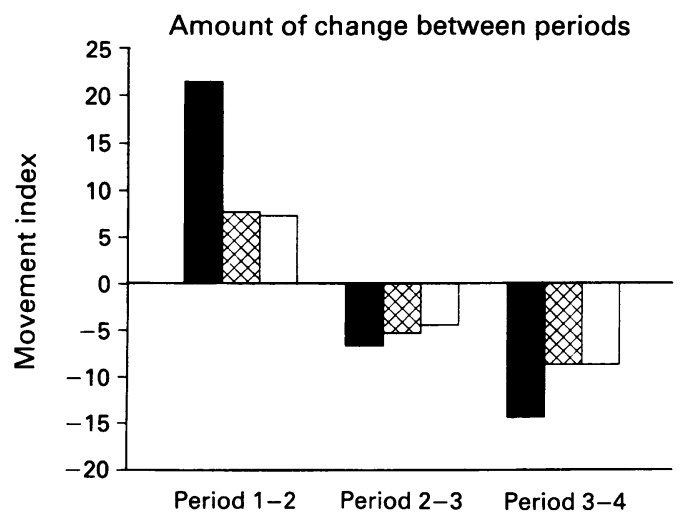

Controls UPDRS Total $51-87$ $\mathrm{H}$ and $\mathrm{Y} 4+5$

and movement index, regardless of the presence or absence of excessive fatigue (not shown).

DIURNAL PATTERN OF THE MOST AFFECTED PATIENT GROUPS

Patient groups with a flattened diurnal pattern were further analysed to determine which periods were responsible for the differences when compared with the controls. For both the activity level and movement index, the most prominent difference when compared with the controls concerned the reduced amount of change between the first and second period (fig 3 ).

\section{Discussion}

We used continuous ambulatory activity monitoring to assess the influence of disease severity on the occurrence of end of day deterioration in PD. Our study confirms the existence of a diurnal pattern of motor activity for both healthy subjects and PD patients. However, regardless of the clinical variable and its severity, we found no evidence of end of day deterioration. In the most severely affected patients, however, we found an almost flat diurnal pattern of motor activity. In these patients there is a conspicuously reduced increase in the activity level and movement index between the first and second morning period. In these patients the designation "depressed morning start" would seem more appropriate. The significance of this altered diurnal pattern is unknown. A flattening of the diurnal pattern may very well be encountered in other chronic diseases.

Two papers remarked on the decline of functional capacity and worsening of Parkinsonian symptoms in the afternoon and evening. ${ }^{45}$ One study found that contrast sensitivity in patients with $P D$ is significantly worse at 1430 than at 0830 , and considered this as evidence of circadian fluctuations parallel to the common pattern of motor variation in PD. ${ }^{15}$ However, contrast sensitivity at 1630 and 0830 showed no differences, and no later measurements were performed. On the other hand, the absence of diurnal changes of responsiveness to levodopa and apomorphine in patients with PD suggests that there are no important diurnal changes in striatal dopaminergic responsiveness. ${ }^{67}$

Excessive fatigue is a serious complaint in PD reported by almost $50 \%$ of patients. Surprisingly few reports have drawn attention to this symptom. ${ }^{45}$ Contrary to previous reports we found no consistent diurnal pattern for fatigue, as most of the patients with fatigue reported no time at which this symptom mainly occurred. In line with these findings is the absence of any influence of excessive fatigue on the diurnal motor activity patterns of PD patients. The similar mean time at which both groups retired to bed, although representing circumstantial evidence, also argues against the previously reported predominant end of day occurrence of fatigue in PD patients. Melatonin is one of many factors that may be associated with fatigue. ${ }^{16} \mathrm{~A}$ study on the circadian rhythmicity of plasma melatonin and urinary 6-hydroxy melatonin sulphate concentrations showed no differences between healthy subjects and treated or untreated PD patients. ${ }^{16}$ Our findings refute an association of fatigue and an intrinsic circadian factor.

This study was supported in part by a grant from Merck Sharp and Dohme, the Netherlands.

1 Renfrew JW, Pettigrew KD, Rapoport SI. Motor activity and sleep duration as a function of age in healthy men. Physiology and Behavior 1987;41:627-34

2 Brown AC, Smolensky MH, D'Alonzo GE, Redman DP. Actigraphy: a means of assessing circadian patterns in human activity. Chronobiology Int 1990;7:125-33.

3 Hilten JJ van, Middelkoop HAM, Kerkhof GA, Roos RAC. A new approach in the assessment of motor activity in Parkinson's disease. F Neurol Neurosurg Psychiatry 1991;54:976-9.

4 Marsden CD, Parkes JD. "On and off" variability and response swings in Parkinson's disease. In: Rose FC, response swings in Parkinson's disease. In: Rose FC, Capildeo R, eds. Research progress in Parkinson's disease,

Marsden CD, Parkes JD, Quin N. Fluctuations of disabilMarsden CD, Parkes JD, Quin N. Fluctuations of disabil-
ity in Parkinson's disease-clinical aspects. In: Marsden CD, Fahn S, eds. Movement disorders. London Butterworth, Scientific 1982: 96-122.

6 Frankel JP, Pirtosek Z, Kempster PA, Bovingdon M, Webster R, Lees AJ, Stern GM. Diurnal differences in response to oral levodopa. $\mathcal{F}$ Neurol Neurosurg Psychiatry 1990;53:948-50.

7 Gancher ST, Nutt JG. Diurnal responsiveness to apomorphine. Neurology 1987;37:1250-3.

8 Folstein M, Folstein S, McHugh PJ. "Mini-Mental State": a practical method for grading the cognitive state of patients for clinicians. $\mathcal{F}$ Psychiat Res 1975;12:189-98.

9 Hoehn MM, Yahr MD. Parkinsonism: onset, progression and mortality. Neurology 1967;17:427-42.

10 Fahn S, Elton RL. Unified Parkinson's Disease Rating Scale. In: Fahn, et al, eds. Recent developments in Parkinson's Disease. New fersey; MacMillan Healthcare, 1987;2:153-63.

11 Montgomery SA, Åsberg M. A new depression scale designed to be sensitive to change. Br f Psychiatry 1979; 134:382-9.

12 McPartland RJ, Kupfer DJ, Foster FG, Reisler KL Mathews G. Objective measurement of human moto activity. Biotelemetry 1975;2:317-23.

13 Borbely AA. New techniques for the analysis of the human sleep-wake cycle. Brain Dev 1986;8:482-8.

14 Armitage P, Berry G. Statistical methods in medical research. Second $\mathrm{P}$, Bition. Statistical methods in medical research. Publications, 1987.

15 Struck LK, Rodnitzky RL, Dobson JK. Circadian fluctuations of contrast sensitivity in Parkinson's disease. tions of contrast sensitivity
Neurology 1990;40:467-70.

16 Critchley PHS, Malcom GP, Malcom PN, Gibb WR Arendt J, Parkes JD. Fatigue and melatonin in Arendt J, Parkes JD. Fatigue and melatonin in Neurosurg Psychiatry 1991;54:91-2. 